\title{
Ischemic stroke after left atrial appendage occlusion with LARIAT in a patient with a coagulation disorder and unrecognized carotid artery stenosis
}

\author{
Krzysztof Bartus ${ }^{1}$, Danuta Sorysz ${ }^{2}$, Radosław Litwinowicz ${ }^{1}$, Boguslaw Kapelak ${ }^{1}$, Artur Dziewierz ${ }^{2}$, \\ Stanislaw Bartus² \\ ${ }^{1}$ Department of Cardiovascular Surgery and Transplantology, Jagiellonian University Medical College, John Paul II Hospital, Krakow, Poland \\ ${ }^{2} 2^{\text {nd }}$ Department of Cardiology and Cardiovascular Interventions, University Hospital, Krakow, Poland
}

Adv Interv Cardiol 2020; 16, 4 (62): 521-523

DOI: https://doi.org/10.5114/aic.2020.101785

A 62-year old man with paroxysmal atrial fibrillation (AF), $\mathrm{CHA}_{2} \mathrm{DS}_{2}$-VASc score 6 and HAS-BLED score 4, with a history of multiple strokes in the right cerebral hemisphere, previous subdural bleeding and subdural hematoma during oral anticoagulation (OAC) requiring surgical craniotomy underwent uneventful atrial appendage occlusion (LAAO) with a LARIAT device (SentreHEART Inc, Redwood, CA) and was discharged home with aspirin monotherapy.

Two and half months later, another ischemic stroke was confirmed by brain computed tomography (CT). No critical stenosis of carotid and vertebral arteries was confirmed by duplex ultrasound (DUS).

Transesophageal echocardiography with contrast was performed to evaluate the heart as a potential source of thrombus. The LAA was fully closed with no signs of recanalization and no thrombus formation was found in the $L A$, LAA neck (stump), or any other heart chambers (Figure $1 \mathrm{~B}$ ).

Despite the multiple negative ultrasound evaluations in the past, $\mathrm{CT}$ angiogram of the carotid arteries was done. A short $(5 \mathrm{~mm})$ critical stenosis not visible in ultrasound in the intracranial right internal carotid artery (RICA) was found (Figure 1 A). Additionally, coagulation marker examination showed increased clot lysis time (CLT) of $121 \mathrm{~min}$, reduced clot permeability $\left(\mathrm{K}_{\mathrm{s}}\right)$ of $6.3 \times 10^{-9} \mathrm{~cm}^{2}$, elevated platelet count of $621 \times 10^{3} / \mu \mathrm{l}$ and a high fibrinogen level of $6.2 \mathrm{~g} / \mathrm{l}$.

In patients with intracranial stenosis aggressive medical treatment is superior to stenting [1]. However, in our case, due to the history of recurrent cerebral bleeding that caused neurological dysfunction and the current high HAS-BLED score, only aspirin monotherapy was con- tinued and the patient was qualified for elective carotid artery stenting.

LAAO is very effective in the reduction of stroke risk in patients with non-valvular AF. Unfortunately, LAAO does not eliminate the thromboembolic risk completely [2]. Thrombus formation can take place in other heart chambers, and coagulation disorders can play a significant role in this process [3]. In contrast to endocardial devices, epicardial occlusion with a LARIAT device leaves no foreign thrombogenic material in the bloodstream and quick epithelialization occurs [4]. In our patient, the LAA was fully closed with no signs of recanalization, and due to negative duplex ultrasound of carotid and vertebral arteries he could be categorized as a low-risk patient. So, why did this patient develop another stroke?

The tests performed just after the stroke excluded heart, as well as carotid and vertebral arteries as the source of thrombus. However, the performance of DUS can be highly variable depending on the operator's experience and lesion localization. Because of intracranial localization of RICA stenosis multiple DUS examinations were negative. Due to the similar location of all the strokes and an increased prothrombotic state, CT angiography was performed, confirming the presence of a critical stenosis in the intracranial RICA on a relatively short lesion. Importantly, carotid CT angiography after a negative duplex ultrasound, as well as coagulation profile assessment, is not routinely performed in patients scheduled for LAAO. Thus, in our opinion, a more detailed coagulation profile and CT scan evaluations might be justified in selected LAAO candidates, especially in cases of stroke recurrence after successful LAA occlusion.

\section{Corresponding author:}

Radosław Litwinowicz MD, PhD, Department of Cardiovascular Surgery and Transplantology, Jagiellonian University Medical College, John Paul II Hospital, Krakow, Poland, phone: +48 1261430 75, e-mail: radoslaw.litwinowicz@uj.edu.pl

Received: 21.04.2020, accepted: 3.06.2020. 


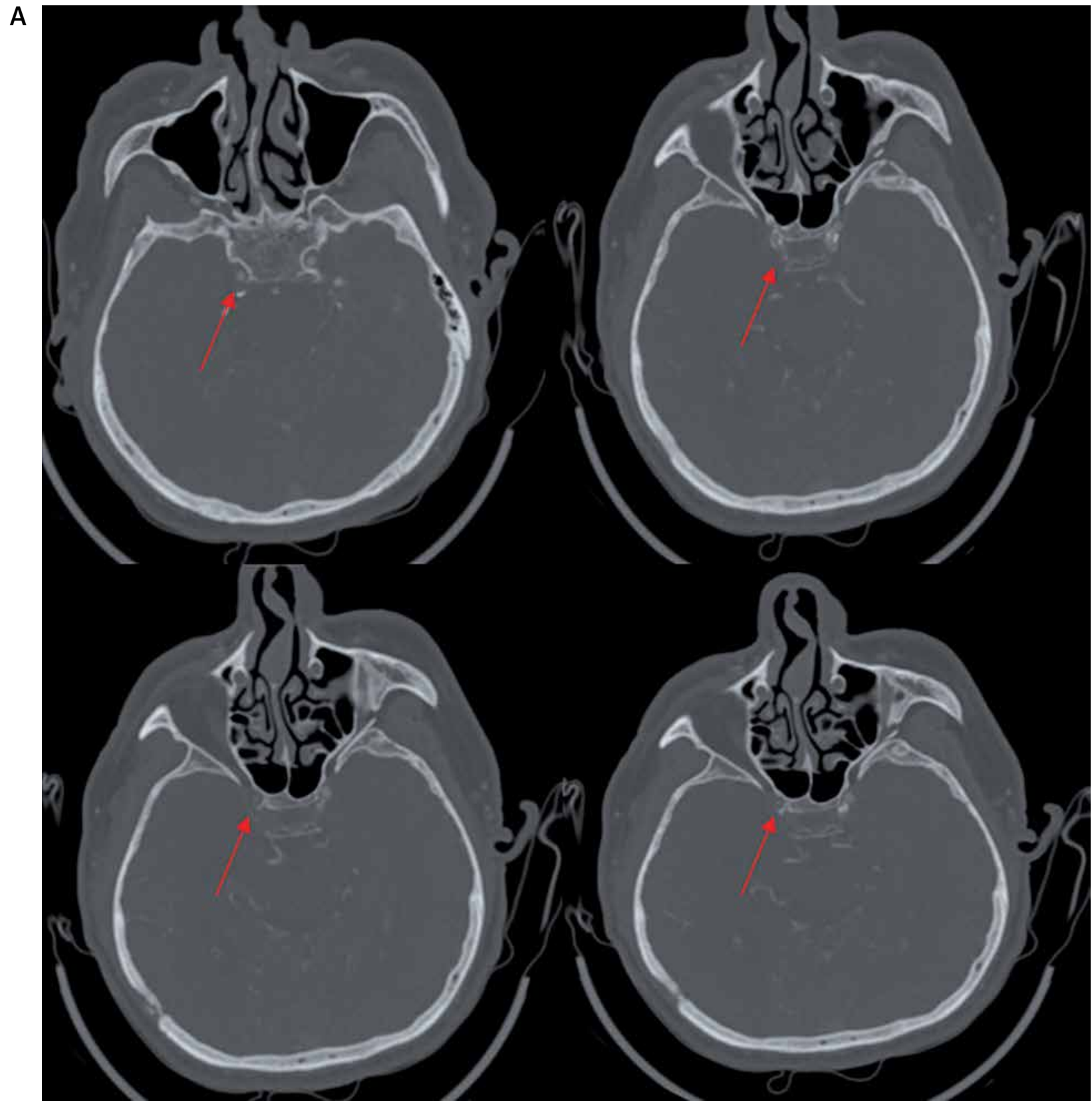

Figure 1. A-Computed tomography angiogram of the carotid arteries - a short $(5 \mathrm{~mm})$ critical stenosis (arrow)

\section{Acknowledgments}

This study is the result of research grant No. UMO2014/13/D/NZ5/01351 funded by the National Science Centre. The funders had no role in study design, data collection and analysis, decision to publish, or preparation of the manuscript.

\section{Conflict of interest}

Bartus $\mathrm{K}$ and his institutions Jagiellonian University Medical College and John Paul II Hospital in Krakow Poland were recipients of the above research grant from the Polish National Science Centre. The other authors declare no conflict of interest. 
B

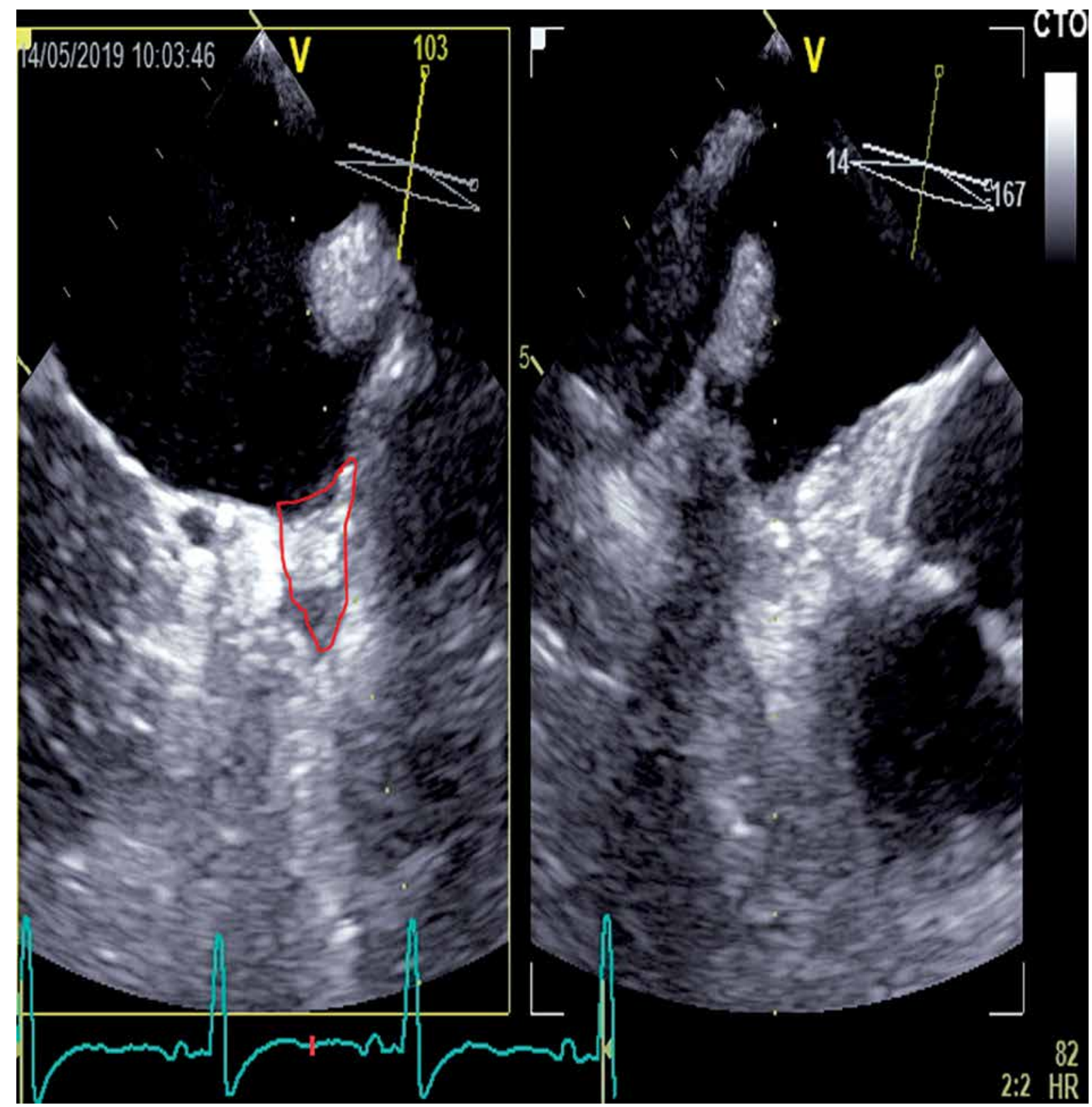

Figure 1. Cont. B - Complete LAA closed with no signs of recanalization (red line - LAA stump). No signs of thrombus formation in LA

\section{References}

1. Derdeyn CP, Chimowitz MI, Lynn MJ, et al. Aggressive medical treatment with or without stenting in high-risk patients with intracranial artery stenosis (SAMMPRIS): the final results of a randomised trial. Lancet 2014; 383: 333-41.

2. Litwinowicz R, Bartus $M$, Kapelak B, et al. Reduction in risk of stroke and bleeding after left atrial appendage closure with LARIAT device in patients with increased risk of stroke and bleeding: long term results. Catheter Cardiovasc Interv 2019; 94: 837-42.

3. Bartus K, Litwinowicz R, Natorska J, et al. Coagulation factors and fibrinolytic activity in the left atrial appendage and other heart chambers in patients with atrial fibrillation: is there a local intracardiac prothrombotic state? (HEART-CLOT study). Int J Cardiol 2020; 301: 103-7.
4. Grygier M, Wojakowski W, Smolka G, et al. Left atrial appendage occlusion: consensus document of association of cardiovascular interventions and heart rhythm section of Polish Cardiac Society. Kardiol Pol 2018; 76: 677-97. 